\title{
Anti-oxidant, anti-inflammatory, and anti-carcinogenic activities of araloside A from Aralia elata
}

\author{
Je-Hyuk Lee ${ }^{1 *}$, Jin-Sun Kim², Seon-Dae Shin ${ }^{1}$ \\ ${ }^{1}$ Department of Food and Nutrition, Kongju National University, Yesan, Korea. \\ ${ }^{2}$ Major in Food and Nutrition, Department of Integrated Life Science and Technology, Kongju National University, Yesan, Korea.
}

\section{ARTICLE INFO \\ Received on: 30/03/2018 \\ Accepted on: 25/09/2018 \\ Available online: $31 / 10 / 2018$}

\section{Key words:}

Anti-inflammation, anticancer, araloside A, Aralia elata.

\begin{abstract}
The aim of this study was to explore the anti-inflammatory and anti-cancer activities of araloside A. Araloside A had a low antioxidant activity. The decrease of 2,2-Diphenyl-1-picrylhydrazyl radicals was only about 5.2\% at 200 $\mathrm{mM}$ of araloside A. Additionally, the araloside A $(200 \mathrm{mM})$ showed approximately $11 \%$ and $9.2 \%$ decrease in $\mathrm{NO}$ radical chemical scavenging activity and superoxide dismutase-like activity, respectively. The estimated oxygen radical absorbance capacity and hydroxyl radical absorbance capacity values of araloside A were approximately $119.7 \mathrm{mM}$ trolox equivalent/g and $0.818 \mathrm{mM}$ gallic acid equivalent/g, respectively. Based on cytotoxicity results, LPS-stimulated (Lipopolysaccharide) macrophage cells were treated with $0-500 \mu \mathrm{M}$ of araloside A, a concentration range that did not reduce the viability of macrophage cells. Araloside A inhibited the production of NO in the macrophages in a concentration-dependent manner. In particular, the $500 \mathrm{mM}$ of araloside A reduced NO production to less than the basal level of NO in macrophage cells. Araloside A showed cytotoxicity against the stomach cancer cell lines, SNU-638 and AGS. Additionally, the melanoma cell line B16-F1 was susceptible to araloside A-mediated cytotoxicity. However, the araloside A did not cause cytotoxicity in the ovary cancer cell line NIH: OVCAR-3. Taken together, our results indicated that the araloside A had the anti-inflammatory activity inhibiting NO production and anti-cancer activity against SNU, AGS cancer cells, and melanoma ovarian cancer cells, in spite of its low antioxidant activity. These results provide the fundamental information for araloside A as an agent against both inflammation and cancer.
\end{abstract}

\section{INTRODUCTION}

The relationship between inflammation and cancer has been recognized for a considerable time but the detailed mechanisms were not understood completely. Chronic inflammatory diseases, autoimmunity, and environmental pollutant/foodborne toxin-causing inflammation stimulate the progress of carcinogenesis (Grivennikov et al., 2010). In addition, the inflammation induces the production of cytokines and the reactive oxygen species/reactive nitrogen intermediates, which cause the initiation of tumor progress through mutation of the cell. Inflammation plays an important role in the pathophysiology of cancers. Inversely, the cancers may cause

\section{"Corresponding Author}

Je-Hyuk Lee, Department of Food and Nutrition, Kongju National University, Yesan, Korea.E-mail: leejeh211@ kongju.ac.kr or intensify inflammation (Díaz et al., 2015). Inflammation and cancer interact with the progress of their pathogenesis each other. For this reason, inflammation and cancer should be considered together in terms of physiological mechanisms, and drugs that have inhibitory effects on both inflammation and cancer are needed.

Various medicinal plants and foods have both anti-inflammatory and anti-cancer activities. Ginger extract (Habib et al., 2008), mushroom extract and its single compounds (Ma et al., 2013), mulberry extract (Eo et al., 2014), and Tripterygium wilfordii (Wong et al., 2012) were reported to have the anti-inflammatory and anti-cancer activities. Aralia elata (A. elata), a medicinal plant for diabetes, hepatics, and stomachache in Korea, China, and Japan (Hu and Wang, 2009), has been investigated for its anti-oxidant activity, anti-cancer activity (Tomatsu et al., 2003), and anti-inflammatory activity (Lee and Jeong, 2009). Araloside A (saponin B, Chikusetsusaponin IV), isolated from $A$. elata, has the anti-gastric and anti-ulcer 
activities against gastric lesions and ulcer by $\mathrm{HCl}$-ethanol and aspirin, respectively (Lee et al., 2005). However, little is known about the anti-inflammatory and anti-cancer activities of araloside A.

The aim of this study was to explore the anti-inflammatory and anti-cancer activities of araloside $\mathrm{A}$ to provide fundamental information for the use of araloside $\mathrm{A}$ in treatments of both inflammation and cancer.

\section{MATERIALS AND METHODS}

\section{Chemical and reagents}

2,2-Diphenyl-1-picrylhydrazyl (DPPH), 2,2'-azino-bis (3-ethylbenzothiazoline-6-sulphonic acid) (ABTS), pyrogallol, sodium nitroprusside, and 2,2'-azobis (2-methylpropionamidin e)-dihydrochloride (AAPH) were obtained from Sigma-Aldrich Inc. (St. Louis, MO). Araloside A [Fig. 1, CAS (Chemical Abstract Service): $7518-22-1, \geq 98 \%$ purity by HPLC (High performance Liquid Chromatography)], which is a Saponin B [Chikusetsusaponin IV, $3 \beta-(4-O-\alpha$-L-arabinofuranosyl- $\beta$-D-glucopyranuronosyloxy) oleana-12-ene-28-oic acid 28- $\beta$-D-glucopyranosyl ester], was obtained from Shanghai Yuanye Bio-Technology, Co. Ltd. (Shanghai, China). Cell culture medium and reagents, such as Dulbecco's modified Eagle medium (DMEM), fetal bovine serum (FBS), penicillin/streptomycin, and trypsin-EDTA (Ethylene Diamine Tetraacetic Acid) were purchased from GIBCO (Invitrogen Inc., Grand Island, NY). Other reagents were used as the first-grade reagents.

\section{Assay for antioxidant activity}

\section{DPPH radical scavenging activity}

Quenching effect of araloside A against DPPH radical was determined by the following procedure: Araloside A $(0.2 \mathrm{ml}$, methanolic solution) was added to $4 \mathrm{ml}$ of methanol and $0.5 \mathrm{ml}$ of $1 \mathrm{mM}$ DPPH. After mixing vigorously for 15 seconds, the mixture left for the reaction at room temperature for 30 minutes. To analyze the scavenging activity of araloside A for DPPH radical, the absorbance of mixtures was measured on $517 \mathrm{~nm}$ using UV-spectrophotometry (Libra S22, Biochrom Ltd., CB4 OFJ, England). Scavenging activity of araloside A for DPPH radical was evaluated through the comparison of absorbance between blank and araloside A.

\section{Reducing capacity}

Reducing capacity was analyzed by araloside A-mediated $\mathrm{Fe}^{3+}$ reduction (Oyaizu, 1986). Araloside A (1 mg/ml) was mixed with $2.5 \mathrm{ml}$ of phosphate buffer $(0.2 \mathrm{M}, \mathrm{pH} 6.6)$ and $2.5 \mathrm{ml}$ of $1 \%$ $\mathrm{K}_{3} \mathrm{Fe}(\mathrm{CN})_{6}$. After the incubation of mixture at $50^{\circ} \mathrm{C}$ for 20 minutes, the reaction mixture was added by $2.5 \mathrm{ml}$ of trichloroacetic acid $(10 \%)$ and centrifuged at $2,090 \times g$ for 10 minutes. A supernatant layer $(2.5 \mathrm{ml})$ was mixed with distilled water $(2.5 \mathrm{ml})$ and $0.1 \% \mathrm{FeCl}_{3}(0.5 \mathrm{ml})$. The reduction of $\mathrm{Fe}^{3+}$ was determined by the absorbance of the mixture at $700 \mathrm{~nm}$ using UV-spectrophotometry (Libra S22, Biochrom Ltd., CB4 OFJ, England).

\section{ABTS radical scavenging activity}

Scavenging activity of araloside A against $\mathrm{ABTS}^{+}$ free radical was measured by a little-modified method of Thaipong et al. (2006). Scavenging against the $\mathrm{ABTS}^{+}$radical was identified using a decolorization reaction (blue-green color) by araloside A. The solution of $7 \mathrm{mM}$ ABTS in phosphate-buffered saline (2.45 mM, pH 7.4) was prepared as a stock and was reacted for 24 hours at room temperature in the dark place for the generation of $\mathrm{ABTS}^{+}$radical. Dark blue-green $\mathrm{ABTS}^{+}$radical solution was diluted using distilled water to an absorbance value of $0.70( \pm 0.02)$ on $732 \mathrm{~nm}$ using a spectrophotometer (SpectraMax M2; Molecular Devices Inc., Sunnyvale, CA). ABTS ${ }^{+}$solution was prepared freshly for each assay. Araloside A $(10 \mu \mathrm{l}, 100 \mathrm{mM})$ was added to $190 \mu \mathrm{l}$ of the $\mathrm{ABTS}^{+}$solution and was allowed to react for 30 minutes in the dark. The decrease of absorbance in reactant was determined on $732 \mathrm{~nm}$ using spectrophotometer (SpectraMax M2; Molecular Devices Inc.). Trolox was used as a standard for the quantification of ABTS decreases.

\section{Superoxide dismutase like activity}

A slight modified method of Marklund and Marklund (1974) was used for the superoxide dismutase (SOD)-like scavenging activity of araloside A. One hundred microliter of araloside A (1 mg/ml), $30 \mathrm{ml}$ of pyrogallol $(7.2 \mathrm{mM})$, and $1 \mathrm{ml}$ of Tris-HCl buffer $(50 \mathrm{mM}, \mathrm{pH} 8.5)$ were mixed and reacted at room temperature for 10 minutes. After 10 minutes of incubation at room temperature, $\mathrm{HCl}$ solution $(50 \mathrm{ml}, 1 \mathrm{M})$ was added for stopping the reactions. Absorbance of the reaction mixture was measured using microplate reader (SPARK 10M, Tecan, Grödig, Austria) on $420 \mathrm{~nm}$, and ascorbic acid (0-1 mM) was used as a positive control.

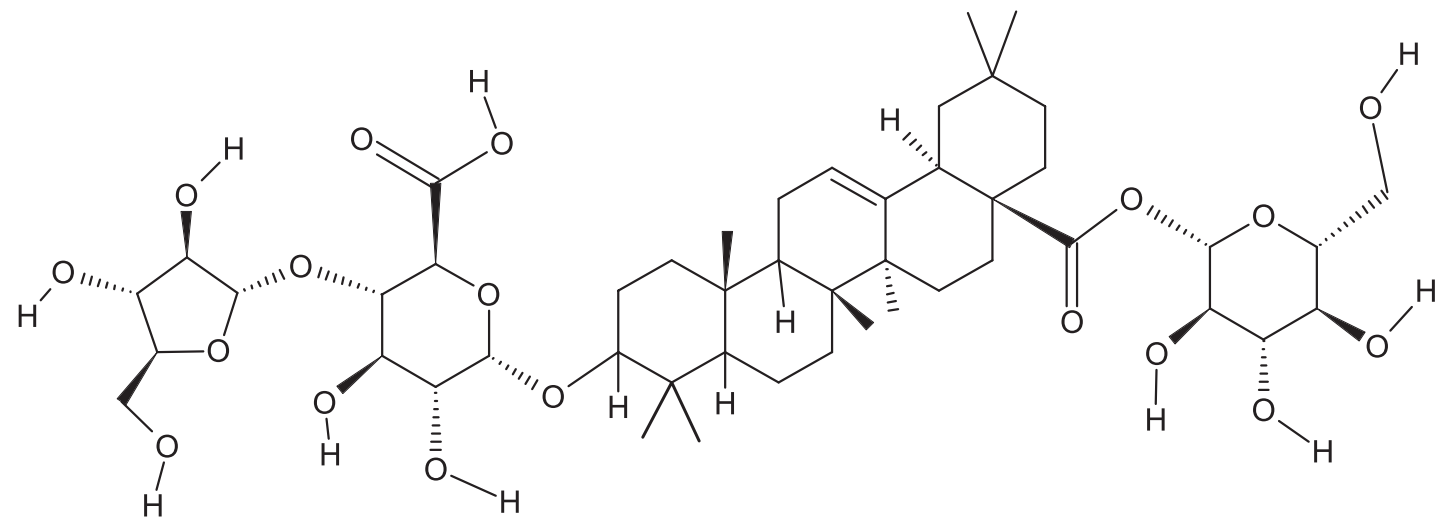

Figure 1. Chemical structure of araloside A. 


\section{Chemical scavenge of nitric oxide radical}

Chemical scavenging activity of araloside A against nitric oxide radical was analyzed by the following method (Babu et al., 2001). Sodium nitroprusside in the aqueous solution of physiological $\mathrm{pH}$ interacts with oxygen and generates $\mathrm{NO}$ radical. Araloside A was added to sodium nitroprusside $(10 \mathrm{mM}$ in PBS) and reacted at $25^{\circ} \mathrm{C}$ for 150 minutes. Then, the same amount of reaction mixture and Griess reagent (1\% sulfanilamide in $5 \%$ phosphoric acid and $0.1 \% \mathrm{~N}-1$-naphthylethylenediamine dihydrochloride in distilled water) were mixed, and the chromophore formed was measured on $540 \mathrm{~nm}$.

\section{Oxygen radical absorbance capacity and hydroxyl radical absorbance capacity}

The oxygen radical absorbance capacity (ORAC) of araloside A was detected by the method of Číž et al. (2010). The ORAC assays monitors the antioxidant scavenging function of araloside A against peroxyl radical induced by AAPH. The antioxidant substance inhibits the loss of fluorescence for fluorescein by the peroxyl radical. One hundred and seventy microliters of fluorescein solution ( $60 \mathrm{nM}$ final concentration) and $10 \mu \mathrm{l}$ of araloside A were added to a well of the microplate (clear bottom, black plate) and were incubated at $37^{\circ} \mathrm{C}$ for 30 minutes. Then, $20 \mu$ of AAPH (50 mM final concentration) was added to each well rapidly to initiate the reaction. The fluorescence of the mixture was recorded every 5 minutes, and the microplate was automatically shaken before reading by an ELISA (Enzyme-linked Immunosorbent Assay) plate reader (Spark 10M, Tecan, Grodig, Austria). The final ORAC values were calculated by a regression equation between the standard antioxidant trolox concentration and the net area under the curve (AUC). The net AUC corresponding to araloside A was calculated by subtracting the AUC corresponding to the blank. ORAC values were expressed as $\mathrm{mg}$ of trolox equivalents (TE)/g dry weight (DW) of araloside A.

The hydroxyl radical absorbance capacity (HORAC) measures the metal-chelating and protection ability activities of araloside A against hydroxyl radicals (Lee and Lee, 2014). Fluorescein $170 \mu \mathrm{l}(60 \mathrm{nM}$, final concentration) and $10 \mu \mathrm{l}$ of araloside A were mixed for 10 minutes in an ELISA plate reader (Spark 10M, Tecan, Grodig, Austria) at $37^{\circ} \mathrm{C}$. Then, $10 \mu \mathrm{l}$ of $\mathrm{H}_{2} \mathrm{O}_{2}(27.5 \mathrm{mM}$, final concentration) and $10 \mu \mathrm{lof} \mathrm{Co}(\mathrm{II})(15.7 \mathrm{mg}$ of $\mathrm{CoF}_{2} \cdot 4 \mathrm{H}_{2} \mathrm{O}$ and $20 \mathrm{mg}$ of picolinic acid in $20 \mathrm{ml}$ of distilled water) were added. Fluorescence of each well in a microplate was recorded every 5 minutes for 1 hour with a shaking. Gallic acid was used for plotting of a standard curve, and the AUC values were calculated in the same manner as for the ORAC assay. The HORAC of araloside A was expressed as milligram of gallic acid equivalents (GAEs)/g DW of araloside A.

\section{Cell lines and culture condition}

Macrophage cells (RAW 264.7) and melanoma B16-F1 [American Type Culture Collection (ATCC) CRL-6323, skin cancer cell line] were purchased from Korean Cell Line Bank (KCLB) and ATCC (ATCC, Rockville, MD), respectively, and was cultured using DMEM with 10\% FBS and penicillin/streptomycin solution (finally, 1 units $/ \mathrm{ml}$ and $1 \mu \mathrm{g} / \mathrm{ml}$ ). SNU-638, AGE cell line (stomach cancer cell line), and adenocarcinoma (ovary cancer cell line, NIH:
OVCAR-3) were obtained from KCLB, and was cultured using RPMI-1640 (Rosewell Park Memorial Institute) medium containing $10 \% \mathrm{FBS}$ and penicillin/streptomycin (finally, 1 units $/ \mathrm{ml}$ and $1 \mu \mathrm{g} /$ $\mathrm{ml}$ ). Cells were cultured in $5 \% \mathrm{CO}_{2}$ humidified incubator at $37^{\circ} \mathrm{C}$.

\section{Cell viability assay}

Cell viability of macrophage cell, melanoma B16-F1, AGS cell, and adenocarcinoma by araloside A was measured by MTT (Methylthiazolydiphenyl-tetrazolium bromide) assay. Cells were cultured in 96-well culture plate (Corning Inc., USA) at $5 \times 10^{4}$ cells/well for 24 hours. After araloside A was added to each well of the 96-well culture plate, the cells were incubated again for 24 hours. MTT solution $(0.5 \mathrm{mg} / \mathrm{ml}$, final concentration) was added to all wells of the culture plate, and the cells were incubated for 4 hours at $37^{\circ} \mathrm{C}$. After discarding all medium from the plates, $100 \mu \mathrm{l}$ of dimethyl sulfoxide was added to each well for dissolution of formazan formed in cells. MTT formazan was detected by a UV-spectrophotometric plate reader (Spark 10M, Tecan, Grodig, Austria) on $540 \mathrm{~nm}$. Viability of cells was defined as the absorbance ratio (expressed as a percentage) of treated cells to untreated cells.

\section{Determination of NO production from LPS-induced macrophage cells}

Macrophage cell was cultured in 96-well culture plate at $5 \times 10^{4}$ cells $/$ well at $37^{\circ} \mathrm{C}$ for 24 hours. LPS $(1 \mathrm{mg} / \mathrm{ml}$, final concentration) was added to each well for the stimulation of $\mathrm{NO}$ production, and then a given concentration of araloside $\mathrm{A}$ was added to the wells in the culture plate. After 24 hours of incubation, the induced NO in macrophage cells was determined quantitatively using the Griess reaction. NG-monomethyl-L-arginine monoacetate (L-NMMA, $10 \mathrm{mM}$ ) was used as a positive control for the inhibition of NO production in macrophage cells (Sutherland et al., 2001).

\section{Statistical analysis}

All experiments were performed at least triplicate and were expressed as a mean \pm standard deviation. Data were analyzed statistically using one-way analysis of variance and Dunnett's multiple comparison for individual comparisons. Results were considered statistically significant when $p$-values were below than 0.05 .

\section{RESULTS AND DISCUSSION}

\section{Antioxidant activity of araloside $A$}

The antioxidant activity of araloside A was evaluated using DPPH/ABTS/NO radical scavenging activity, reducing capacity, and ORAC/HORAC assay (Table 1). Araloside A was found to have a low antioxidant activity. The DPPH radical scavenging activity of araloside A increased in a treatment concentration-dependent manner although the decrease of DPPH radicals was only about $5.2 \%$ at $200 \mathrm{mM}$ of araloside A. Additionally, the araloside A (200 $\mathrm{mM}$ ) showed approximately $11 \%$ and $9.2 \%$ decrease, as compared to the control, in NO radical chemical scavenging activity and SOD-like activity, respectively. The estimated ORAC and HORAC values of araloside A were approximately $119.7 \mathrm{mM} \mathrm{TE} / \mathrm{g}$ and $0.818 \mathrm{mM} \mathrm{GAE} / \mathrm{g}$, respectively. Araloside A had no reducing capacity or ABTS radical chemical scavenging activity. 
Table 1. Antioxidant activity of araloside A.

\begin{tabular}{lcccc}
\hline \multicolumn{4}{c}{ Araloside A $(\boldsymbol{\mu M})$} \\
\hline Antioxidant assay & 0 & 50 & 100 & 200 \\
DPPH radical scavenging activity & $100.2 \pm 2.65^{\mathrm{a}}$ & $99.9 \pm 5.75^{\mathrm{a}}$ & $97.7 \pm 0.07^{\mathrm{a}}$ & $94.8 \pm 3.10^{\mathrm{a}}$ \\
Reducing capacity & $\mathrm{ND}^{6}$ & $\mathrm{ND}$ & $\mathrm{ND}$ & $\mathrm{ND}$ \\
ABTS radical scavenging activity & $\mathrm{ND}$ & $\mathrm{ND}$ & $\mathrm{ND}$ & $\mathrm{ND}$ \\
NO radical scavenging activity $^{2}$ & $100.0 \pm 1.06^{\mathrm{a}}$ & $88.0 \pm 1.65^{\mathrm{b}}$ & $88.8 \pm 1.59^{\mathrm{b}}$ & $89.0 \pm 0.26^{\mathrm{b}}$ \\
SOD-like activity $^{3}$ & $100.0 \pm 0.99^{\mathrm{a}}$ & $92.2 \pm 1.76^{\mathrm{b}}$ & $89.7 \pm 0.81^{\mathrm{c}}$ & $90.8 \pm 1.10^{\mathrm{b}, \mathrm{c}}$ \\
ORAC $^{4}$ & & $119.7 \pm 9.44 \mathrm{mM} \mathrm{TE} / \mathrm{g} \mathrm{dw}$ & \\
HORAC $^{5}$ & & $0.818 \pm 0.04 \mathrm{mM} \mathrm{GAE} / \mathrm{g} \mathrm{dw}$ & \\
\hline
\end{tabular}

All values are \%control, except of ORAC and HORAC. ${ }^{1}$ Concentration of DPPH radical is $100 \mu \mathrm{M} .{ }^{2} \mathrm{NO}$ radical is $10 \mathrm{mM}$ SNP (sodium nitroprusside). ${ }^{3}$ Pyrogallol $(0.182 \mathrm{mM})$ was used for SOD-like activity. ${ }^{4}$ ORAC: oxygen radical absorbance capacity. ${ }^{5} \mathrm{HORAC}$ : hydroxyl radical absorbance capacity. ${ }^{6} \mathrm{ND}$ : Not detected. Values followed by the different letter in the same column are significantly different $(p<0.05)$.

This study is the first report to describe the low level of antioxidant activity for araloside A. Generally, the compounds with physiological activities such as anti-inflammation and anti-cancer tend to have potent anti-oxidative activities (Reuter et al., 2010). Although a potent antioxidant activity in extracts or compounds is expected to be associated with their significant anti-inflammatory and anti-cancer activities, it does not necessarily guarantee this characteristic. Cai et al. (2004) reported that among 112 Chinese medical herbs with anti-cancer activity, approximately $43.8 \%$ of herbs had a weak antioxidant activity and only $18.7 \%$ of herbs had a high anti-oxidant activity. Additionally, the flavonoid gnaphaliin and pinocembrin had a much lower DPPH radical scavenging activity and superoxide anion scavenging activity compared to tiliroside. However, the anti-inflammatory activities of gnaphaliin and pinocembrin were similar or slightly better than those of tiliroside for mouse paw edema induced by phospholipase $\mathrm{A}_{2}$ or serotonin, for ear edema induced by TPA-induced ear edema, and for sheep red blood cell-induced delayed hypersensitivity (Sala et al., 2003).

\section{Inhibition of NO production in LPS-stimulated macrophage cells by araloside $A$}

The cytotoxicity of araloside A against macrophage cells was investigated in a preliminary study by determining the level of inhibition of NO production in LPS-stimulated macrophage cells; NO production is one of the major biomarkers for inflammatory activity. No cytotoxicity against macrophage cells was found in $0-500 \mu \mathrm{M}$ of araloside A; however, 1,000 $\mu \mathrm{M}$ of araloside A showed approximately $17 \%$ cytotoxicity (Fig. $2 \mathrm{~A}$ ). Based on this result, we selected the $0-500 \mu \mathrm{M}$ concentration range of araloside A for subsequent experiments, as this would not affect the viability of the macrophage cells.

NO production in macrophage cells was stimulated significantly from 0.8 to $46.3 \mu \mathrm{M}$ by addition of LPS (Fig. 2B). NG-methyl-L-arginine (NMMA, $10 \mu \mathrm{M}$ ), an inhibitor for inducible nitric oxide synthase (iNOS), suppressed NO production to $16.3 \mu \mathrm{M}$ (35.2\%, \% control) in LPS-stimulated macrophage cells. Araloside A $(25-500 \mathrm{mM})$ was added to LPS-stimulated macrophage cells and found to inhibit production of NO in a concentration-dependent manner. Treatment with 50 and $100 \mathrm{mM}$ of araloside A resulted in $2.8 \%$ and $11.7 \%$ inhibition of $\mathrm{NO}$ production, respectively. Especially, $500 \mathrm{mM}$ of araloside A inhibited significantly NO production to less than basal level of NO in macrophage cells.
Anti-inflammatory suppression of NO production in LPS-stimulated macrophage cells by saponins was reported by several researchers. Saponins from Tacca vietnamensis and Panax ginseng inhibited significantly NO production, expression of related enzymes, and production of various cytokines, which are involved in the anti-inflammatory process (Jang et al., 2016). The oleanolic acid 3-O-beta-dglucopyranosyl $(1 \rightarrow 3)$-alpha-l-rham-nopyranosyl $(1 \rightarrow 2)$-alpha-1arabinopyranoside, a triterpenoid saponin in $A$. elata, has been shown to inhibit LPS-induced NO production by down-regulating production of the NF- $\kappa \mathrm{B}$ protein complex in macrophage cells (Suh et al., 2007). Based on these result, we suggest that the antiinflammatory activity of araloside A (saponin B) acts through inhibition of NO production in LPS-induced macrophage cells.

\section{Cytotoxicity of araloside A against cancer cell lines}

Cell viability of various cancer cell lines by treatment of araloside A was investigated, as shown in Figure 3. Araloside A had a cytotoxic effect against the stomach cancer cell lines SNU-638 and AGS. The SNU cells showed the dose-related cell death with approximately $62 \%$ and $92 \%$ of cell death by the addition of 100 and $200 \mathrm{mM}$ araloside A, respectively. No cytotoxicity was observed in AGS cells after addition of 50 $\mathrm{mM}$ araloside A, but $13 \%$ and $92 \%$ cell death was shown by the treatment of 100 and $200 \mathrm{mM}$ araloside A, respectively. Additionally, melanoma had a susceptive cytotoxicity in 100-200 mM of araloside A and showed approximately $96 \%$ of cytotoxicity in $200 \mathrm{mM}$ of araloside A. By contrast, the ovary cancer cell line NIH: OVCAR-3 did not show any cytotoxicity after treatment with $0-200 \mathrm{mM}$ of araloside A. Taken together, araloside A varied in its toxicity depending on cancer cell lines; especially had a potent cytotoxicity against stomach cancer cell lines (SNU and AGS cells).

Saponins, isolated from A. elata, showed anti-cancer activity against human leukemia cancer cells (HL60), human lung cancer cells (A549), and human prostatic cancer cells (DU145) (Zhang et al., 2012). The anticancer activity of araloside $\mathrm{A}$ in this study was consistent with the previous reports. Several compounds from herbal extracts had both anti-cancer and anti-inflammatory activities, and the related mechanisms were elucidated. Ergosterol peroxide/trametenoic acid from Inonotus obliquus suppressed the activity of NF-kB and production of NO significantly and provided the significant 
A
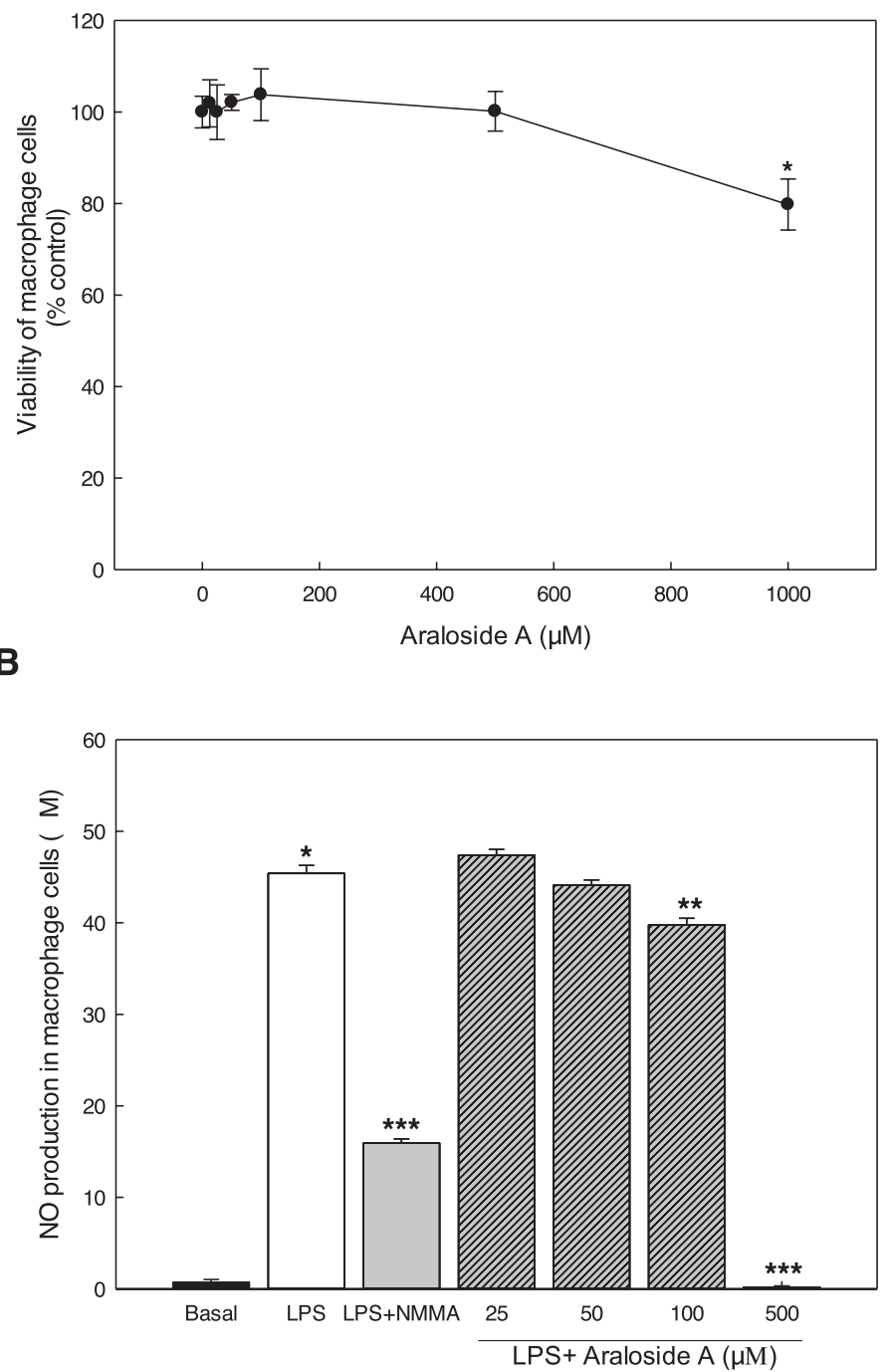

Figure 2. Cell viability and inhibition of NO production from LPS-induced macrophage cells by araloside A. Viability of macrophage cells by araloside A. * $p<0.001$, compared to the control; $\mathrm{B}:{ }^{*} p<0.001$, compared to the basal; ${ }^{* *} p<0.05$ and ${ }^{* * *} p<0.001$, compared to the LPS-treated group.

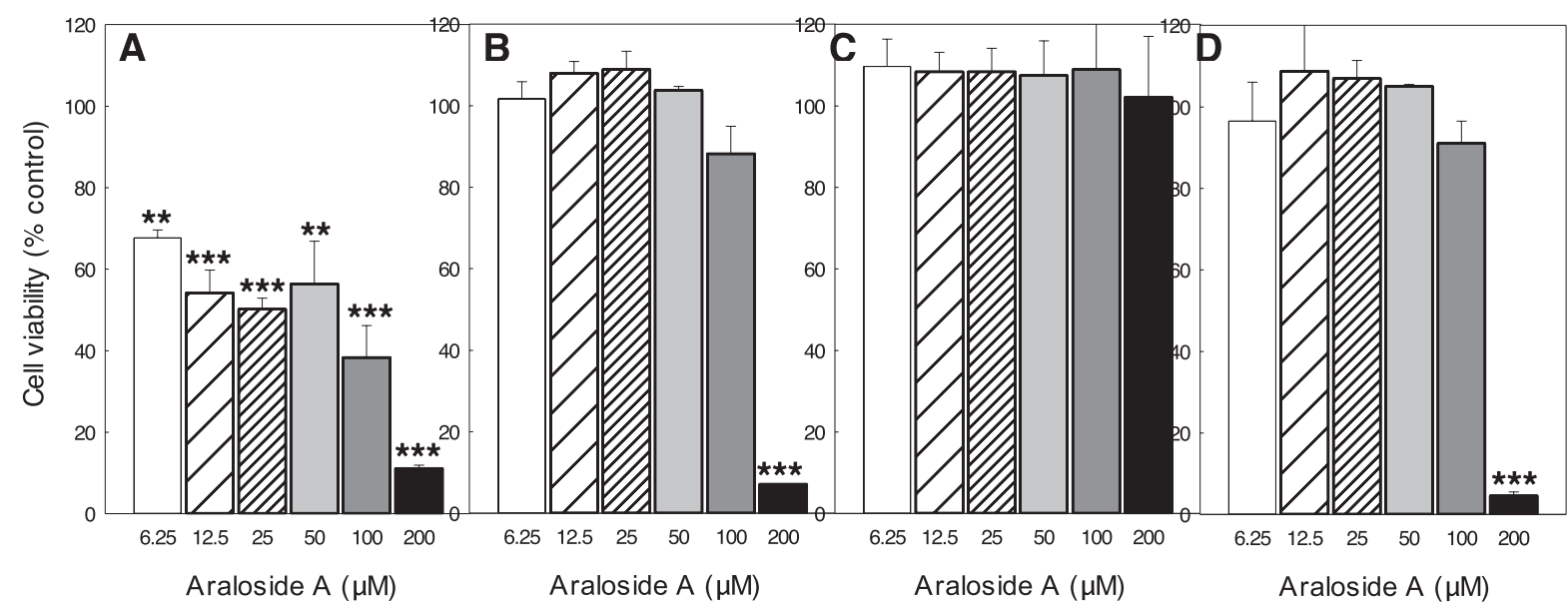

Figure 3. Anti-carcinogenic activity of araloside A. (A) SNU: stomach cancer cell (control $=99.99 \% \pm 15.24 \%$ ), (B) AGS: stomach cancer cell (control $=100 \% \pm 9.15 \%),\left(\right.$ C) Melanocyte: skin cancer cell (control $=100.04 \% \pm 8.46 \%$ ), and (D) SK-OV-3: ovary cancer cell $($ control $=100.28 \% \pm 9.90 \%),{ }^{*} p<0.005$ and $* * * p<0.001$, compared to the control. 
cytotoxicity against the breast cancer cells (Ma et al., 2013). Thus, many single compounds had both anti-inflammatory and anti-cancer activities, and it has been proved scientifically in an animal study, as well as cellular level.

Cancers are associated with inflammation, and the expression of inflammatory genes has an inverse correlation with the progress of cancers and their prognosis. Production of NO was reported to contribute to carcinogenesis, and the uncontrolled overproduction of NOcaused the improved duplication of oncogenes and oxidative damage in DNA. It has been well known that the proinflammatory mediators contribute to carcinogenesis and progress of cancers. NF-kB, a pro-inflammatory cytokine, activates the oncogenes and surviving genes, such as cyclin D1 and $c-m y c$ (Zhu et al., 2001). Actually, NF-kB was active constitutively in clinical samples of the patients with multiple myelom, lung cancer, and pancreatic cancer (Tang et al., 2006; Dhillon et al., 2008). Patients with colitis infection-caused inflammation have a high risk for the progression of colon cancers (Itzkowitz and Yio, 2004), and chronic inflammation due to Helicobacter pylori makes patients vulnerable to some cancers (Peter and Beglinger, 2007). Additionally, the anti-inflammatory agents, such as nonsteroidal anti-inflammatory drugs, had a role as therapeutic agents for cancers (Coussens and Werb, 2002). These various reports indicated a clear relationship between anti-inflammatory activity, including the suppression of NO production, and cytotoxicity against cancer cell lines. It will be necessary to elucidate the more detailed mechanisms between anti-inflammatory and anti-cancer mechanisms.

\section{CONCLUSION}

Araloside A had anti-inflammatory activity inhibiting NO production, and anti-cancer activity against SNU, AGS cancer cells, and ovarian cancer cells. This study demonstrated the potential health-enhancing activity of araloside A due to its inhibition of both inflammation and cancer in spite of its low anti-oxidative activity.

\section{ACKNOWLEDGMENTS}

This work was supported by the research grant of the Kongju National University in 2015.

\section{CONFLICT OF INTEREST}

There is no conflict of interest.

\section{REFERENCES}

Babu BH, Shylesh BS, Padikkala J. Antioxidant and hepatoprotective effect of Acanthus Ilicifolius. Fitoterapia, 2001; 72:272-7.

Cai Y, Luo Q, Sun M, Corke H. Antioxidant activity and phenolic compounds of 112 traditional Chinese medicinal plants associated with anticancer. Life Sci, 2004; 74:2157-84.

Číž M, Čížová H, Denev P, Kratchanova M, Slavov A, Lojek A. Different methods for control and comparison of the antioxidant properties of vegetables. Food Control, 2010; 21:518-23.

Coussens LM, Werb Z. Inflammation and cancer. Nature, 2002; 420:860-7.

Dhillon N, Aggarwal BB, Newman RA, Wolff RA, Kunnumakkara AB, Abbruzzese JL, Ng CS, Badmaev V, Kurzrock R. Phase II trial of curcumin in patients with advanced pancreatic cancer. Clin Cancer Res, 2008; 14:4491-9.
Díaz L, Díaz-Muñoz M, García-Gaytán AC, Méndez I. Mechanistic effects of calcitriol in cancer biology. Nutrients, 2015; 7:5020-50.

Eo HJ, Park JH, Park GH, Lee MH, Lee JR, Koo JS, Jeong JB. Anti-inflammatory and anti-cancer activity of mulberry (Morus alba L.) root bark. BMC Complement Altern Med, 2014; 14:200-8.

Grivennikov SI, Greten FR, Karin M. Immunity, inflammation, and cancer. Cell, 2010; 140:883-99.

Habib SH, Makpol S, Abdul Hamid NA, Das S, Ngah WZ, Yusof YA. Ginger extract (Zingiber officinale) has anti-cancer and antiinflammatory effects on ethionine-induced hepatoma rats. Clinic, 2008; 63:807-13.

$\mathrm{Hu}$ WC, Wang MH. Antioxidant activity of Aralia elata seeds fractions. Hortic Environ Biotechnol, 2009; 50:253-7.

Itzkowitz SH, Yio X. Inflammation and cancer IV. Colorectal cancer in inflammatory bowel disease. The role of inflammation. Am J Physiol Gastrointest Liver Physiol, 2004; 287:G7-17.

Jang KJ, Choi SH, Yu GJ, Hong SH, Chung YH, Kim CH, Jeong JB. Anti-inflammatory potential of total saponins derived from the roots of Panax ginseng in lipopolysaccharide-activated RAW 264.7 macrophages. Exp Ther Med, 2016; 11:1109-15.

Lee JH, Jeong CS. Suppressive effects on the biosynthesis of inflammatory mediators by Aralia elata extract fractions in macrophage cells. Environ Toxicol Pharmacol, 2009; 28:333-41.

Lee EB, Kim OJ, Kang SS, Jeong C. Araloside A, an antiulcer constituent from the root bark of Aralia elata. Biol Pharm Bull, 2005 28:523-6.

Lee JI, Lee JH. Antioxidant and inhibitory activities of thioflavanones against nitric oxide production. Food Sci Biotechnol, 2014 3:957-63.

Ma L, Chen H, Dong P, Lu X. Anti-inflammatory and anticancer activities of extracts and compounds from the mushroom Inonotus obliquus. Food Chem, 2013; 139:503-8.

Marklund S, Marklund G. Involvement of the superoxide anion radical in the autoxidation of pyrogallol and a convenient assay for superoxide dismutase. Eur J Biochem, 1974; 47:469-74.

Oyaizu M. Studies on product of browning reaction: antioxidative activities of products of browning reaction prepared from glucosamine. Jap J Nutr, 1986; 44:307-15.

Peter S, Beglinger C. Helicobacter pylori and gastric cancer: the causal relationship. Digestion, 2007; 75:25-35.

Reuter S, Gupta SC, Chaturvedi MM, Aggarwal BB. Oxidative stress, inflammation, and cancer: how are they linked? Free Radic Biol Med, 2010; 49:1603-16.

Sala A, Recio MC, Schinella GR, Máñez S, Giner RM, CerdáNicolás M, Ríos JL. Assessment of the anti-inflammatory activity and free radical scavenger activity of tiliroside. Eur J Pharmacol, 2003; 461:53-61.

Suh SJ, Jin UH, Kim KW, Son JK, Lee SH, Son KH, Chang HW, Lee YC, Kim CH. Triterpenoid saponin, oleanolic acid 3-O-betad-glucopyranosyl(1®3)-alpha-1-rhamnopyranosyl(1@2)-alpha-1arabinopyranoside (OA) from Aralia elata inhibits LPS-induced nitric oxide production by down-regulated NF-kappaB in raw 264.7 cells. Arch Biochem Biophys, 2007; 467:227-33

Sutherland H, Khundkar R, Zolle O, Mcardle A, Simpson AWM, Jarvis JC, Salmons S. A fluorescence-based method for measuring nitric oxide in extracts of skeletal muscle. Nitric Oxide, 2001; 5:475-81.

Tang X, Liu D, Shishodia S, Ozburn N, Behrens C, Lee JJ, et al. Nuclear factor-kappaB (NF-kappaB) is frequently expressed in lung cancer and preneoplastic lesions. Cancer, 2006; 107:2637-46.

Thaipong K, Boonprako U, Crosby K, Cisneros-Zevallos L, Byrne DH. Comparison of ABTS, DPPH, FRAP, and ORAC assays for estimating antioxidant activity from guava fruit extracts. J Food Composit Anal, 2006; 19:669-75. 
Tomatsu M, Ohnishi-Kameyama M, Shibamoto N. Aralin, a new cytotoxic protein from Aralia elata, inducing apoptosis in human cancer cells. Cancer Lett, 2003; 199:19-25.

Wong KF, Yuan Y, Luk JM. Tripterygium wilfordii bioactive compounds as anticancer and anti-inflammatory agents. Clin Exp Pharmacol Physiol, 2012; 39:311-20.

Zhang Y, Ma A, Hu C, Wang L, Le L, Song S. Cytotoxic triterpene saponins from the leaves of Aralia elata. Fitoterapia, 2012; 83:806-11.

Zhu L, Fukuda S, Cordis G, Das DK, Maulik N. Anti-apoptotic protein survivin plays a significant role in tubular morphogenesis of human coronary arteriolar endothelial cells by hypoxic preconditioning. FEBS Lett, 2001; 508:369-74.

How to cite this article:

Lee J-HL, Kim J-S, Shin S-D. Anti-oxidant, anti-inflammatory, and anti-carcinogenic activities of araloside A from Aralia elata. J App Pharm Sci, 2018; 8(10): 129-135. 